\title{
XIX. Heating effects at the anode in vacuum-tubes
}

\section{B. Hodgson M.Sc. Ph.D.}

To cite this article: B. Hodgson M.Sc. Ph.D. (1914) XIX. Heating effects at the anode in vacuumtubes, Philosophical Magazine Series 6, 27:157, 189-202, DOI: $10.1080 / 14786440108635077$

To link to this article: http://dx.doi.org/10.1080/14786440108635077

曲 Published online: 08 Apr 2009.

Submit your article to this journal

Џll Article views: 2

Q View related articles $₫$ 
based on a calculation of the actual value of the diffusion coefficient of hydrogen atoms through ordinary hydrogen. The present results show that the actual degree of dissociation is much smaller than that previously found, and that even at $3500^{\circ} \mathrm{K}$. hydrogen is probably not dissociated to a very great extent. It is hoped that the experiments at very low pressures will make possible a quantitative estimation of the degree of dissociation.

In conclusion, the writer wishes to point out that the value of 130,000 calories referred to by Bohr is undoubtedly too higb, and that the experimental evidence now indicates a value of about 75,000 to 80,000 calories. That the correct value, however, is as low as the 60,000 calculated by Bohr seems very improbable.

XIX. Heating Effects at the Anode in Vacuum-Tubes. By B. Hodgson, M.Sc., Ph.D., Assistant Leeturer in Physics, The University, Bristol*.

$\$ 1$. T $\mathrm{N}$ a previous communication the anthor showed 1 that the energy communicated to the anode during discharge in air was approximately proportional to the current through the tube. The experiments were made with gas-pressures $2 \cdot 7,1 \cdot 05$, and $0.63 \mathrm{~mm}$. The experiments to be described have been carried out over a large range of currents and pressures in oxygen. The apparatus used was that described in earlier papers $\ddagger$. The method of experimenting was slightly altered. Instead of allowing the anode to rise to that temperature at which the loss of energy by radiation was equal to the gain from the bombardment of negative carriers, the rise in temperature in a definite interval of time was measured and a correction for loss by radiation applied. This method was found to yield results much more quickly with only a small loss in accuracy. The anode used was of lead, and had a heat capacity of $2 \cdot 2 \mathrm{gm}$. calories.

\section{§2. Eaperimental Results.}

The results in Table $I$. show the heat energy received by the anode in one second for currents varying from $0.001 \mathrm{amp}$. to $0.007 \mathrm{amp}$. and pressures between $3.8 \mathrm{~mm}$. and

* Communicated by the Autbor.

$\uparrow$ Hodgson, Phil. Mag. xxv. p. 458 (1913).

t Hodgson, Phil. Mag. xxv. p. 453 (1913): Hodgson \& Mainstone, Phil. Mag. xxvi. p. 411 (1913). 
$0.18 \mathrm{~mm}$. of mercury. The quantities given yield ergs if multiplied by $10^{4}$.

TABLE I.

\begin{tabular}{|c|c|c|c|c|c|c|c|}
\hline$i$ alups. & $\cdot 001$ & 002 & .003 & .004 & .005 & .006 & $00 \pi$ \\
\hline \multicolumn{8}{|l|}{ Pressure } \\
\hline $\begin{array}{c}\operatorname{mm} . \\
3.8\end{array}$ & $\ldots$ & 75 & 90 & 124 & 146 & 197 & 222 \\
\hline $3 \cdot 08$ & & 75 & 110 & 132 & 163 & & 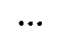 \\
\hline $2 \cdot 25$ & 35 & 73 & 102 & 131 & 172 & 211 & \\
\hline $1 \cdot 5$ & 70 & 138 & 194 & 258 & 312 & 362 & 402 \\
\hline $1 \cdot 33$ & 69 & 128 & 186 & 248 & 299 & 346 & 399 \\
\hline $1 \cdot 21$ & 66 & 120 & 181 & 234 & 289 & 349 & 418 \\
\hline 1.10 & 63 & 115 & 154 & 217 & 272 & 321 & 364 \\
\hline 1.00 & 63 & 110 & 166 & 200 & 266 & 311 & 356 \\
\hline 0.81 & 56 & 102 & 152 & 199 & 249 & 289 & 348 \\
\hline $0 \cdot 60$ & 49 & 96 & 135 & 190 & 220 & 260 & 281 \\
\hline 0.50 & 48 & 90 & 119 & 150 & 145 & 157 & 127 \\
\hline 0.41 & 38 & 69 & 69 & 50 & 44 & 59 & 96 \\
\hline 0.35 & 34 & 17 & 28 & 43 & 74 & 121 & 330 \\
\hline 0.28 & 12 & 21 & 61 & 196 & 576 & 一 & - \\
\hline 0.23 & 27 & 218 & & & & & \\
\hline $0 \cdot 18$ & 83 & & & & & & \\
\hline
\end{tabular}

The corresponding values of the potential (V) between the electrodes of the vacuum-tube are given in volts in Table II.

'TABLE II.

\begin{tabular}{|c|c|c|c|c|c|c|c|}
\hline$i \operatorname{annps.}$ & $\cdot 001$ & .002 & $\cdot 003$ & .004 & 005 & .006 & .007 \\
\hline $\begin{array}{c}\text { Pressure } \\
\text { mm. } \\
3 \cdot 8 \\
3.08 \\
2 \cdot 25 \\
1 \cdot 5 \\
1 \cdot 33 \\
1 \cdot 21 \\
1 \cdot 10 \\
1 \cdot 00 \\
0.81 \\
0 \cdot 60 \\
0.50 \\
0 \cdot 41 \\
0.35 \\
0.28 \\
0 \cdot 23 \\
0 \cdot 18\end{array}$ & $\begin{array}{c}630 \\
560 \\
660 \\
647 \\
620 \\
617 \\
612 \\
597 \\
663 \\
627 \\
672 \\
750 \\
940+ \\
1700 \S \\
2750 \$\end{array}$ & $\begin{array}{c}565 \\
527 \\
530 \\
655 \\
632 \\
627 \\
627 \\
625 \\
635 \\
660 \\
720 \\
777 \\
885 \\
1260+ \\
31008\end{array}$ & $\begin{array}{c}515 \\
505 \\
520 \\
660 \\
652 \\
632 \\
635 \\
642 \\
655 \\
702 \\
787 \\
855^{*} \\
100{ }^{*} \\
1560 \$\end{array}$ & $\begin{array}{c}502 \\
490 \\
530 \\
650 \\
647 \\
647 \\
647 \\
643 \\
675 \\
740 \\
848 \\
940^{*} \\
1210^{\dagger} \\
2200 \$\end{array}$ & $\begin{array}{c}490 \\
487 \\
527 \\
650 \\
635 \\
650 \\
650 \\
632 \\
680 \\
777 \\
872^{*} \\
1075 \dagger \\
1300 \dagger\end{array}$ & $\begin{array}{c}490 \\
517 \\
542 \\
652 \\
640 \\
647 \\
655 \\
637 \\
680 \\
807 \\
950^{*} \\
1230^{\circ} \\
-\end{array}$ & $\begin{array}{c}510 \\
- \\
640 \\
642 \\
650 \\
657 \\
640 \\
680 \\
830 \\
980^{*} \\
15008 \\
-\end{array}$ \\
\hline
\end{tabular}


These potential differences were measured by means of a Braun electrometer. The asterisks and other signs following some of these quantities denote the type of discharge occurring in the tube. With high pressures there appears on the anode, the anode glow which is succeeded by a dark space and the faintly luminous positive column. In this case no distinguishing sign follows the values of $V$. Then as either the current increases or the pressure decreases, the positive column contracts towards the anode till only the surface of the anode is illuminated. This condition is denoted by the asterisk *. Then as the pressure decreases further, the anode glow disappears, and except for the negative glow, no luminosity appears in the tube. This condition occurred only once-with a current of $0.002 \mathrm{amp}$., and a pressure of $0.35 \mathrm{~mm}$. As the vacuum becomes more perfect, there appears a hazy luminosity throughout the tube, without any glow on the anode. This condition is denoted by the sign $\dot{\dagger}$. Finally, the luminosity in the tube increases in brightness, and there is cast behind the anode a shadow, which becomes more definite as the pressure is reduced. The sign $\S$ marks this stage.

Tables III. and IV. give other results for the energy ( $M \theta$ ) given to the anode per second for constant current $0.003 \mathrm{amp}$, at various pressures.

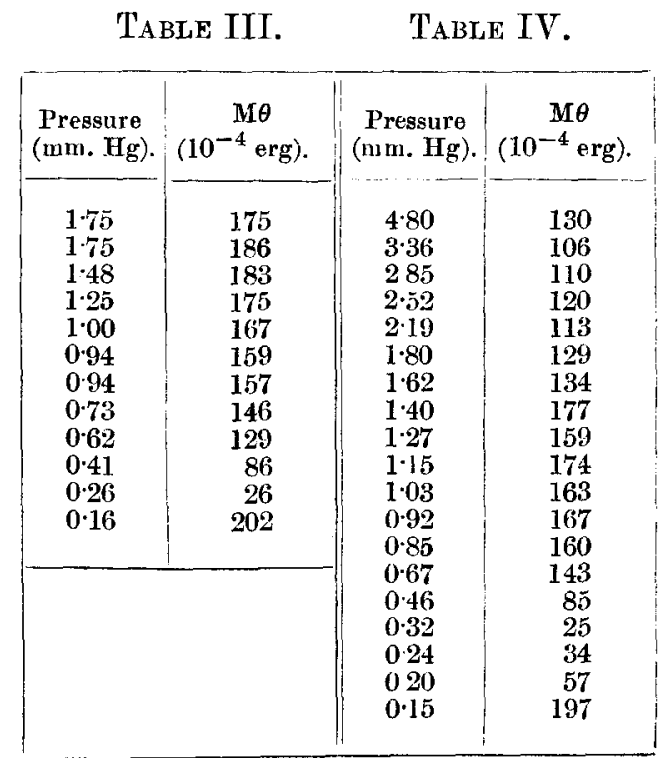


Fig. 1 shows four results taken from Table I. Three of the curves are moved bodily upwards for the sake of clearness. Energy supplied to the anode per second is plotted Fir. I.

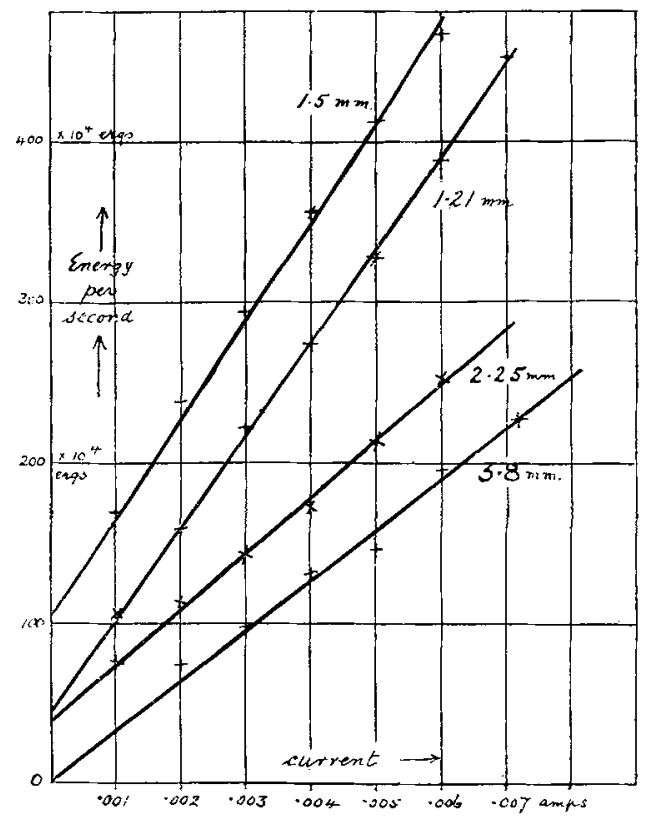

against current for constant pressure. A linear relation exists at the pressures used. For lower pressures the curves are of a different type, and fig. 2 gives some of these, which all possess the same general shape. For small currents proportionality exists between current and energy, and then as the current increases the energy communicated to the anode actually decreases and falls to a minimum value, after which a quick rise is obtained. For currents smaller than 0.001 amp. no values were obtained, but the probable courses for the $0.28 \mathrm{~mm}$. and the $0.35 \mathrm{~mm}$. curves are shown by the dotted parts. 'The curves in fig. 1 would probably show the same form could currents large enough be obtained.

If certain assumptions be mate, the average energy per ion can be obtained. If $n$ negative carriers with only one charge strike the anode per second and do not ionize there, then $n e=i$, where $i$ is the current and $e$ the charge per ion. Then the energy given to the anode per second is $n e V^{\prime}$ where $V^{\prime}$ is the mean potential through which the ion falls, and

$$
n e \mathrm{~V}^{\prime}=i \mathrm{~V}^{\prime} \text {. }
$$


the Anode in Vacuum-Tubes.

Fig. 2.

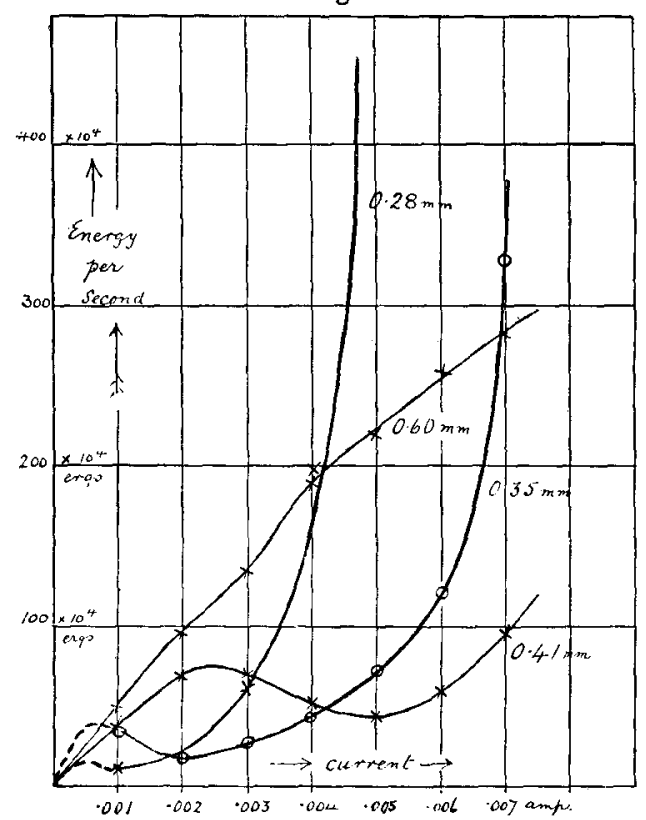

If, then, the results in Table I. are divided by the current the average voltage fall per ion is obtained. This is given in volts in the following table:-

TABLE V.

\begin{tabular}{|c|c|c|c|c|c|c|c|}
\hline$i$ amps. & $\cdot 001$ & $\cdot 002$ & $\cdot 003$ & $\cdot 004$ & .005 & .006 & $\cdot 007$ \\
\hline \begin{tabular}{|c|} 
Pressure. \\
(mm.). \\
3.80 \\
$3 \cdot 08$ \\
$2 \cdot 25$ \\
$1 \cdot 50$ \\
$1 \cdot 33$ \\
$1 \cdot 21$ \\
$1 \cdot 10$ \\
$1 \cdot 00$ \\
$0 \cdot 81$ \\
$0 \cdot 60$ \\
$0 \cdot 50$ \\
$0 \cdot 41$ \\
$0 \cdot 35$ \\
$0 \cdot 28$ \\
$0 \cdot 23$ \\
$0 \cdot 18$
\end{tabular} & $\begin{array}{l}59 \\
55 \\
35 \\
70 \\
69 \\
66 \\
63 \\
63 \\
56 \\
49 \\
48 \\
38 \\
34 \\
12 \\
27 \\
83\end{array}$ & $\begin{array}{r}37 \\
37 \\
36 \\
69 \\
64 \\
60 \\
58 \\
55 \\
51 \\
48 \\
45 \\
35 \\
8 \\
10 \\
109\end{array}$ & $\begin{array}{r}33 \\
37 \\
34 \\
65 \\
62 \\
61 \\
51 \\
56 \\
51 \\
45 \\
40 \\
23 \\
9 \\
20\end{array}$ & $\begin{array}{l}31 \\
33 \\
33 \\
65 \\
62 \\
59 \\
54 \\
50 \\
50 \\
45 \\
38 \\
12 \\
11 \\
49\end{array}$ & $\begin{array}{r}29 \\
33 \\
34 \\
63 \\
55 \\
58 \\
55 \\
53 \\
50 \\
44 \\
29 \\
8 \\
15 \\
115\end{array}$ & $\begin{array}{l}33 \\
33 \\
35 \\
60 \\
58 \\
58 \\
54 \\
52 \\
48 \\
43 \\
26 \\
10 \\
20\end{array}$ & $\begin{array}{l}- \\
\\
57 \\
57 \\
60 \\
52 \\
51 \\
50 \\
40 \\
18 \\
14 \\
47\end{array}$ \\
\hline
\end{tabular}

Phil. Mag. S. 6. Vol. 27. No. 157. Jan. 1914. 
If now $V^{\prime}$ is plotted against pressure with constant current the curves shown in fig. 3 are obtained. They show also the

Fig. 3.

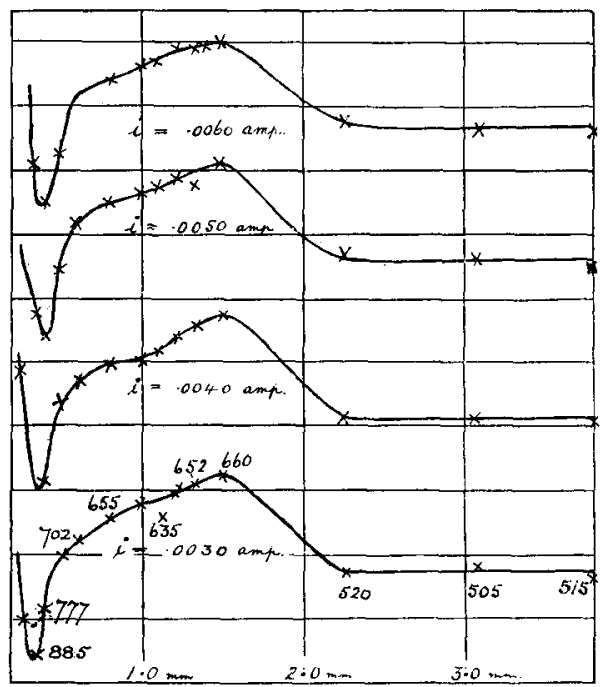

relation between pressure and energy received per second, the scale being in this case different for each curve. Five additional curves, similar in shape, can be got from Tables III., IV., and V. They all show a pressure range from 2 to $4 \mathrm{~mm}$. in which the energy given to the anode is approximately constant. As the pressure is reduced the energy per ion increases to a maximum at about $1.5 \mathrm{~mm}$. pressure. It then falls to a minimum whose position and value vary with the current, and after this minimum a rapid rise is obtained for lower pressures.

\section{§3. Theoretical Conclusions.-T. The Large Heating Effect.}

From Table I. it will be seen that the energy communicated to the anode per second for a current of 0.003 amp. was practically constant and equal to $100 \times 10^{4} \mathrm{ergs}$ for pressures between 2 and $4 \mathrm{~mm}$. This is equivalent to an average ionic energy of 35 volts (Table V.), assuming that the ions do not ionize at the anode surface. It is easy to show that this energy cannot be acquired by the ion in its mean free path. The anode fall is about 20 volts, and this fall occurs quite close to the anode, so close that a sound 
cannot be got near enough to the anode to measure the distance in which this voltage drop occurs. Now the mean free path of an electron in oxygen at $3 \mathrm{~mm}$. pressure is about $1.4 \times 10^{-2} \mathrm{~cm}$., and as the potential gradient in the positive column is small, the maximum energy that an electron can acquire in moving up to the anode from a point distant $1.4 \times 10^{-2} \mathrm{~cm}$. is the energy of the anode fall at most, i.e. a 20-volt energy. As the measured energy is 35 volts, a 15-volt energy must be accounted for in some other way.

Now a rough value of the potential gradient in the positive column can be got from a knowledge of the cathode fall, the anode fall, the potential across the tube, and the distance between the electrodes. In the case considered, the mean gradient in the positive column

$$
\begin{aligned}
& =\frac{515-370 \text { volt }}{10} \frac{\mathrm{cm} .}{} \\
& =15 \frac{\mathrm{volt}}{\mathrm{cm} .}
\end{aligned}
$$

If, then, we assume that all the energy acquired by the negative carriers in moving up to the anode from a distance of $1 \mathrm{~cm}$. is given up as heat, all the 35 -volt energy is accounted for.

Now in $1 \mathrm{~cm}$. at this pressure, a corpuscle makes about 50 collisions and an ion of molecular size about 200 , so that in spite of a great number of collisions energy is " accumulated" by the negative carriers in some way and transferred to the anode. It is not suggested that all the energy acquired by the carriers within $1 \mathrm{~cm}$. of the anode is transferred to the anode, and none from the positive column beyond this distance. Probably the whole column of gas up to the negative glow contributes to the 15 -volt energy not accounted for by the anode fall.

\section{Possibility of an "Accumulation" of Energy.}

The following considerations show the possibility of an "accumulation" of energy by an ion in spite of frequent collisions.

One possibility-that ionization occurs on collision-may be regarded as very improbable, because the gradient in the positive column is small,

A further possibility is that an aggregate ion or cluster is formed on collision. There is abundant evidence to show 
that clusters and multiple-charged atoms both exist. H. A. Wilson found that the mobility of ions in flames changed suddenly at a certain temperature, suggesting a change in the clustering of the ions. Todd * has proved, from measurements of the mobility of the positive ion at fairly low pressures, the existence of clusters up to cluster 6 in Kleeman's notation $\dagger$, and also $\ddagger$ that the negative ion consists of a cluster which becomes less complex at lower pressures.

Lattey and Tizard $\S$ have obtained evidence of the existence of a cluster of 25 molecules round a positive charge.

Kleeman || has shown from theoretical considerations that there probably exists in ionized gases a continual forming and breaking up of clusters of different orders so that a state of equilibrium is finally reached, in which the number of clusters of different orders depends on the electric field and the gas pressure.

Now it can be shown that the formation of clusters permits the "accumulation" of energy. Consider a cluster $n$, i. e. $n$ molecules aggregated round one charge, colliding with another molecule and forming a cluster $n+1$, the collision being direct. Let the mass, energy of motion, and velocity of cluster $n$ just before collision be respectively

$$
n m, \mathrm{E}_{n} \text { and } v_{n} \text {, }
$$

and let these quantities for cluster $n+1$ immediately after collision be $\overline{n+1} m, \mathrm{E}_{n^{\prime}}$, and $v_{n^{\prime}}$.

Then

or

$$
n m v=\overline{n+1} m v_{n^{\prime}}
$$

$$
v_{n^{\prime}}=\frac{n}{n+1} v_{n} \text {. }
$$

Hence

$$
\mathrm{E}_{n^{\prime}}=\frac{1}{2} \overline{n+1} m\left(\frac{n}{n+1}\right)^{2} v_{n}^{2} \text {. }
$$

Now

therefore

$$
\mathrm{E}_{n}=\frac{1}{2} n \cdot m \cdot v_{n}^{2},
$$

$$
\mathrm{E}_{n^{\prime}}=\frac{n}{n+1} \mathbf{E}_{n}
$$

That is the energy of motion of the cluster just after

* Todd, Phil. Mag. xxv. p. 165̃ (1913).

$\dagger$ Kleeman, Proc. Camb. Phil. Soc. xvi. p. 285 (1912).

\pm Todd, Proc. Camb. Phil. Soc. xvi. p. 21 (1912).

\$ Lattey \& Tizard, Proc. Roy. Soc. lxxxvi. A. p. 356 (1912).

I| Kleeman, Proc. Camb. Phil. Soc. xvi. p. 285 (1912). 
collision is $\frac{n}{n+1}$ of its energy immediately before, and the larger the colliding cluster the smaller the fraction of energy not appearing as energy of motion. The latter fraction, $\frac{1}{n+1} \mathrm{E}_{n}$, probably appears as internal energy of the cluster. In some such way as above indicated, the energy is absorbed by the cluster till such amount is accumulated as to render it unstable. Now if it be assumed that the energy acquired per mean free path is the same for all clusters, an approximate value of the energy of eluster $n$ after the $n$th collision can be shown by induction to be $\mathrm{E}_{n}=\frac{n+1}{2} \mathrm{E}_{1}$ where $\mathrm{E}_{1}$ is the energy gained per mean free path. Now in the case considered above, a 15-volt energy has to be "accumulated," that is, all the energy available in a path $1 \mathrm{~cm}$. long. As this would involve at least 100 collisions, and imply the formation of a cluster 100 , it is improbable that the explanation of the phenomena is as simple as above described. It is clear that, in addition to collisions between clusters and molecules. there will be collisions among the clusters themselves. Once a cluster is formed, its acceleration in the electric field is less than that of the simple ion. So that it is possible for an ion or small cluster to overtake a larger one. In this case combination may take place resulting in a neavier cluster with increased energy, or two clusters if the resulting combination happened to be unstable. The accumulation of energy by the clusters will then reach some limit whicn depends on the number of complex ions of different orders in equilibrium under the conditions of the experiment. In addition to the energy of motion, some fraction of the energy absorbed by the clusters will also be given up to the electrode as heat.

Another explanation of the "accumulation" of energy suggests itself. The charged ion moving towards the anode gains energy in its mean free path, so that after collision with a neutral molecule, the latter rebounds with a greater momentum than it would possess if no current were flowing. This would be repeated after the ion had moved again in the electric field, so that there would be a transference of momentum along the tube towards the anode. This would mean that both a pressure and temperature gradient would exist in the positive column, and on this explanation the excess energy given to the anode would be accounted for by a transference of energy to the anode from a hotter gas surrounding it. 


\section{\$4. Discussion of fig. 3 Curves.}

These curves can be analysed into five distinct parts as shown in the diagram below.

The energy given up to the anode is constant for pressures above $2 \mathrm{~mm}$. as shown in part 1 of fig. 4 . At $2 \mathrm{~mm}$. the energy begins to increase and reaches a maximum at a pressure of $15 \mathrm{~mm}$. for all curves. The potential gradient

Fig. 4.

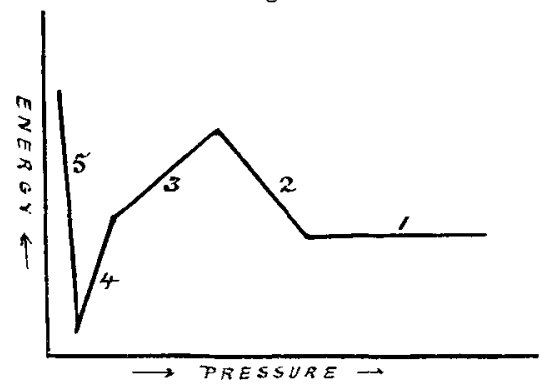

in the tube has also risen. In both parts 1 and 2 of fig. 4 the factor determining the energy appears to be the mean gradient in the tube, for in part 1 both gradient and energy are constant and a fixed ratio appears to hold between gradient and energy for points on parts 1 and 2. This is shown in Table Vl. from results collected from Tables I. and II.

\section{TABLE VI.}

\begin{tabular}{|c|c|c|c|c|}
\hline$i$. & $p r$. & Energy. & Gradient. & Gradient/Energy. \\
\hline$\cdot 002$ & $\left\{\begin{array}{l}3 \cdot 8 \\
1 \cdot 5\end{array}\right.$ & $\begin{array}{r}75 \\
138\end{array}$ & $\begin{array}{l}195 \\
285\end{array}$ & $\left.\begin{array}{l}2 \cdot 6 \\
2 \cdot 1\end{array}\right\}$ \\
\hline$\cdot 003$ & $\left\{\begin{array}{l}3.8 \\
1.5\end{array}\right.$ & $\begin{array}{r}99 \\
194\end{array}$ & $\begin{array}{l}145 \\
290\end{array}$ & $\left.\begin{array}{l}1 \cdot 5 \\
1.5\end{array}\right\}$ \\
\hline$\cdot 004$ & $\left\{\begin{array}{l}3.8 \\
1.5\end{array}\right.$ & $\begin{array}{l}124 \\
258\end{array}$ & $\begin{array}{l}132 \\
280\end{array}$ & $\left.\begin{array}{l}1 \cdot 1 \\
1 \cdot 1\end{array}\right\}$ \\
\hline .005 & $\left\{\begin{array}{l}3.8 \\
1.5\end{array}\right.$ & $\begin{array}{l}146 \\
312\end{array}$ & $\begin{array}{l}120 \\
280\end{array}$ & $\left.\begin{array}{c}0.82 \\
0.90\end{array}\right\}$ \\
\hline$\cdot 006$ & $\left\{\begin{array}{l}3.8 \\
1.5\end{array}\right.$ & $\begin{array}{l}197 \\
362\end{array}$ & $\begin{array}{l}120 \\
282\end{array}$ & $\left.\begin{array}{l}0.61 \\
0.78\end{array}\right\}$ \\
\hline$\cdot 007$ & $\left\{\begin{array}{l}3.8 \\
1.5\end{array}\right.$ & $\begin{array}{l}222 \\
402\end{array}$ & $\begin{array}{l}140 \\
270\end{array}$ & $\left.\begin{array}{l}0.63 \\
0.67\end{array}\right\}$ \\
\hline
\end{tabular}


This implies that the ionization processes are constant over parts 1 and 2, the change in energy being due to changes in mobility of the ions and perhaps in the clustering.

By referring to Table II. it is seen that the voltage across the tube was always about 660 volts at $1.5 \mathrm{~mm}$. pressure when the curve changes abruptly. The voltages actually registered were $660,650,660,650,652$, and 640 volts. The sharp change in the curve is probably occasioned by the commencement of ionization in the positive column. The constant voltage across the tube at this point also suggests that the gradient has become large enough to effect ionization by collision. This ionization reduces the anode energy in two ways. In the first place ions are now travelling in both directions, and collisions can reduce considerably the energy of those moving towards the anode, and in the second place the bombarding negative ions may combine with the free positive ions and cease to have any acceleration in the electric field. Additional evidence of ionization is shown by the decrease in "resistance" of the tube. The potential across the tube is slightly below that in the initial part of section 3 (fig. 4). (For actual values of the potential across the tube see Table II.)

Part 4 (fig. 4) commences at a pressure of $0.5 \mathrm{~mm}$. At this point the energy diminishes rapidly, probably owing to ionization at the anode surface. Here the current is no longer represented by $i=n e$,

but by

$$
i=n e+n_{1} e,
$$

where $n_{1}$ is the number of positive carriers produced per second at the anode; so that the number of bombarding ions is reduced from

$$
n \text { or } \frac{i}{e} \text { to } \frac{i}{e}-n^{\prime},
$$

which number reaches a minimum when $n=n_{1}$, $i$.e. when each negative ion sets free a positive ion on striking the anode. It is, then, possible for the lessening number of bombarding ions, aided by the motion of positive ions in the opposite direction, to give up less energy to the anode in spite of the increased gradient. When the minimum number of negative ions is obtained, further reduction in pressure produces an increase in the gradient in the tube, and hence an increase in the energy given up. This part is represented by the sharply rising part 5 (fig. 4 ).

These hypotheses lead to a fuller explanation of the discharge in vacuum-tubes. Suppose the gas pressure is large, 
say 3-4 $\mathrm{mm}$. in oxygen, and the current small enough to give a normal cathode fall. Although ionization is necessary at the cathode surface to make the current continuous *, the author $\dagger$ has shown that only a small fraction of the bombarding ions produce fresh ions at the cathode surface. The relatively few corpuscles produced soon acquire energy in the cathode dark space sufficient to ionize many times. This energy is expended in the process of ionization in the cathode dark space and the negative glow. The corpuscles produced in the dark space soon produce fresh ions there, and so a progressive ionization occurs as the negative glow is approached. The increase in the number of ions per $\mathrm{cm}$. reduces the gradient, and in the negative glow the "resistance" of the gas there is so small, that the gradient is negligible compared with that in the dark space; and when the electrons or negative ions have expended the energy acquired in the catbode dark space, no further ionization occurs. The formation of clusters begins, and these negative carriers drift in the electric field towards the anode. The hazy glow at the anode may be the result of ionization, but this must be due to only a small fraction of the bombarding ions. Otherwise the shape of the curve in fig. 4 is difficult to account for. As the pressure is reduced the phenomena occur as already described.

The curves in fig. 2 are explained in a similar way to those in fig. 3, as they represent the facts of Tables I. and II. is a different form, namely, with constant pressure instead of constant current.

\section{\$5. Effect of Distance between the Electrodes on the Ener(y! given to the Anode and Cathode.}

A tube was arranged so that one electrode was movable. The distance between the electrodes could be varied between 0 and $30 \mathrm{~cm}$, and this process could be performed by using a winding arrangement without impairing the vacuum.

Though it was not possible to decide the relative values of the two suggestions in $\S 3$, the results are not in discord with either view. During the experiment the current and gas pressure were kept constant, and the distance between the electrodes and the energy given up to cathode or anode were measured. In the case of the cathode, the energy given up was independent of the length of the positive column except

* J. J. Thomson, 'Conduction through Gases,' ii. ed. p. 585.

+ Hodgson, Phil. Mag. xxvi. p. 460 (1913). 
when the distance between the electrodes was so small that the nearness of the cool anode kept the cathode temperature low. With the anode the energy given up increased continuously with the length of the positive column.

The results are shown in the following Table:-

\section{TABLE VII.}

Lead Cathode in Oxygen.

$i=\cdot 0030 \mathrm{amp}$. $p r=2 \cdot 8 \mathrm{~mm}$. Hg.

$\mathrm{L}=$ distance between electrcdes (cm.).

$\theta=$ rise in temperature per minute $\left(0^{\circ} \mathrm{C}\right.$.).

\begin{tabular}{|c|c|c|c|}
\hline L. & $\theta$. & I. & $\theta$. \\
\hline $\begin{array}{l}0 \cdot 3 \\
0 \cdot 3 \\
0 \cdot 65 \\
0 \cdot 65 \\
0 \cdot 70 \\
0 \cdot 70 \\
1 \cdot 80 \\
1 \cdot 80\end{array}$ & $\begin{array}{l}6 \cdot 15 \\
6 \cdot 20 \\
6 \cdot 50 \\
6 \cdot 85 \\
6 \cdot 80 \\
6 \cdot 50 \\
6 \cdot 90 \\
7 \cdot 05\end{array}$ & $\begin{array}{l}8 \cdot 10 \\
10 \cdot 7 \\
13 \cdot 8 \\
17 \cdot 5 \\
22 \cdot 2 \\
25 \cdot 8 \\
29 \cdot 8\end{array}$ & $\begin{array}{l}7 \cdot 15 \\
7 \cdot 00 \\
7 \cdot 10 \\
6 \cdot 90 \\
7 \cdot 05 \\
7 \cdot 10 \\
7 \cdot 02\end{array}$ \\
\hline
\end{tabular}

TABLE VIII.

Lead Anode in Oxygen.

$i=\cdot 0050 \mathrm{amp}$. $p r=3 \cdot 6 \mathrm{~mm} . \mathrm{Hg}$.

\begin{tabular}{|c|c|}
\hline L. & $\theta$. \\
\hline $24 \cdot 1$ & $14 \cdot 2$ \\
$20 \cdot 1$ & $14 \cdot 1$ \\
$16 \cdot 5$ & $14 \cdot 0$ \\
$5 \cdot 9$ & $12 \cdot 6$ \\
$4 \cdot 0$ & $11 \cdot 8$ \\
$2 \cdot 5$ & $10 \cdot 8$ \\
$1 \cdot 3$ & $11 \cdot 8$ \\
\hline
\end{tabular}

$\theta$ here represents the equilibrium temperature of the anode under the above conditions, $i . e$. the temperature the anode rises to after a long interval of time. This method is more sensitive, for the rise in temperature per minute is only 
small. The results of Tables VII. and VIII. are shown graphically in fig. 5. Fig. 5.

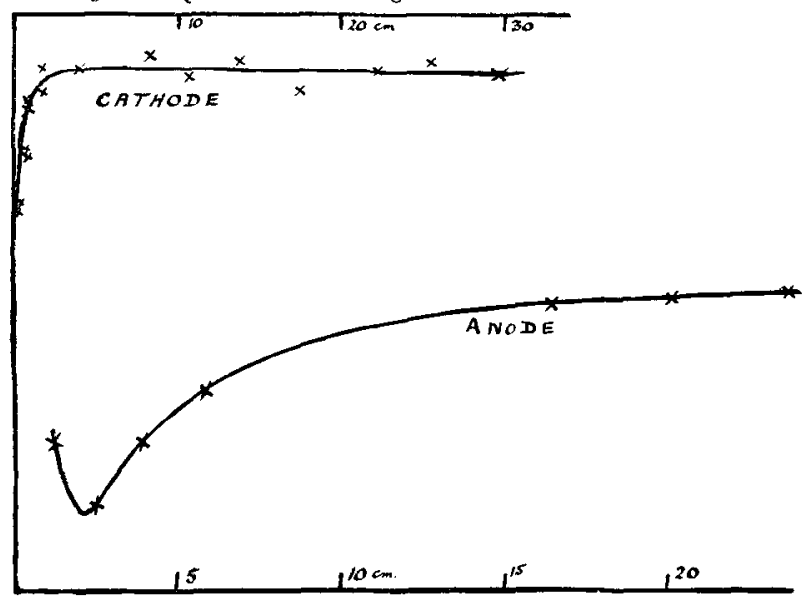

In both curves with short distances, a disturbance is introduced due to the nearness of the electrodes. Neglecting these distortions, it is clear that the energy given up to the cathode is independent of the length of the distance between the electrodes. This agrees with the assumption made before that the bulk of the positive ions originate in the negative glow. Were any produced in the space nearer the anode, the energy given up would increase with the distance between the electrodes.

In the case of the anode an increase in the distance between the electrodes is accompanied by an increase in energy. Though the curves obtained showed a tendency to reach a maximum, none was reached even with a length of discharge of $24 \mathrm{~cm}$. This seems to indicate that the transference of momentum by collision plays a large part in the "accumulation" of energy, for one would expect an equilibrium stage in clustering to be reached long before all the collisions in a $20 \mathrm{~cm}$. path have occurred.

\section{Summary.}

1. Results showing the relation between current, gas pressure, and energy, given to the anode in oxygen have been obtained.

2. The relation between the energy communicated to either electrode and their distance has been investigated.

3. The mechanism of discharge has been discussed in the light of these results.

Pbysics Department,

The University, Bristol. 